30(5), 719-731

\title{
Effects of maximum speed limit on Gyeongbu Expressway
}

\author{
Yinhua Song $^{a} \cdot$ Byeongchan Seong $^{a, 1}$ \\ ${ }^{a}$ Department of Applied Statistics, Chung-Ang University \\ (Received July 27, 2017; Revised August 19, 2017; Accepted August 21, 2017)
}

\begin{abstract}
In September 2010, the Korea government increased the speed limit on the Gyeongbu Expressway (Cheonan IC.-Yangjae IC) from 100 to $110 \mathrm{~km}$ per hour. This paper considers ARIMA-Intervention model to analyze the effects of the speed limit change on the incidences of traffic accidents and injuries. In addition, in order to investigate the effects more clearly, we also analyze the difference between the two lines of Cheonan ICYangjae IC and Busan IC-Cheonan IC. As a result, we observe that the numbers of accidents and injuries have increased after the speed limit change. The increases are strikingly distinctive in comparison to other lines (Busan IC-Cheonan IC) where there have been no changes in the maximum speed limit.
\end{abstract}

Keywords: intervention analysis, ARIMA model, traffic accidents, traffic injuries

\section{1. 서론}

한국도로공사에서는 2010 년 9 월부터 경부선 천안IC-양재IC 구간의 최고 제한속도를 $100 \mathrm{~km} / \mathrm{h}$ 에서 $110 \mathrm{~km} / \mathrm{h}$ 로 상향 조정한다고 발표하였다. 이는 도로여건의 개선 및 자동차성능 향상 등에도 불구하고 연간 81 만건의 운전자들이 과속으로 단속되는 현실을 감안하여 안전운행에 무리가 없고 도로여건이 허 용하는 범위 내에서 운전자들이 수용할 수 있는 합리적인 제한속도를 제시한 것이었다.

한국도로공사에서 발간한 제한속도 상향 시범운영효과 용역분석 보고서에서는 2009년 9월부터 2011년 2 월까지의 교통사고자료를 대상으로 교통량이 반영된 사고율과 사고심각도지수를 이용하여 교통사고 특성을 분석하였다. 그 결과, 제한속도 상향 전 사고율 평균은 7.5 , 제한속도 상향 후 사고율 평균은 7.0으로 나타났고 부산방향의 사고심각도지수는 57.2 로서 상향 전 93.6 보다 낮게 나타났다. 일부 구 간(양재-판교, 신갈-수원)을 제외한 대부분 구간의 사고율과 사고심각도지수는 감소한 것으로 나타나 교통사고의 발생에 악영향을 미치지 않는 것으로 판단하였으며, 다만 장기적인 분석이 필요하다고 판단 하였다 (Lee, 2011).

국외에서 제한속도 조정과 교통사고에 관한 주요한 연구는 1995년 미국의 National Maximum Speed Law (NMSL) 제한속도 통제가 폐지된 후 제한속도 조정으로 인한 지역별 교통사고의 영향을 분석

This research was supported by Basic Science Research Program through the National Research Foundation of Korea (NRF) funded by the Ministry of Education (NRF-2016R1D1A1B03934857).

${ }^{1}$ Corresponding author: Department of Applied Statistics, Chung-Ang University, 84 Heukseok-ro, Dongjakgu, Seoul 06974, Korea. E-mail: bcseong@cau.ac.kr 
하기 위하여 다수 이루어졌다. Ossiander과 Cummings (2002)는 미국 워싱턴 주에서 제한속도가 $89 \mathrm{~km} / \mathrm{h}$ 에서 $105 \mathrm{~km} / \mathrm{h}$ 로 상향된 고속도로를 대상으로 사고충돌 및 차량속도에 관한 연구를 진행하였으 며, 그 결과 제한속도 상향 이후 평균 차량속도는 $5.5 \mathrm{mph}$ 증가하였고 치명적인 충돌사고 발생률은 2 배 이상 증가하였음을 발견하였다. Friedman 등 (2007)은, 1993 년 이스라엘 정부에서 총 $115 \mathrm{~km}$ 의 3 개 주요 고속도로의 제한속도를 $90 \mathrm{~km} / \mathrm{h}$ 에서 $100 \mathrm{~km} / \mathrm{h}$ 로 조정하였을 때, 그 상향정책이 교통사고에 미친 영향을 개입모형으로 평가하였다. 1988 년부터 1999 년의 자료를 분석한 결과, 제한속도 상향조정시 사 망자는 4.69배 증가하고 치사율은 2.5배 증가하였다고 결론을 내렸다. Farmer (2016)는 1993년부터 2013 년의 자료를 이용하여 미국의 최고 제한속도 증가에 따른 안전성 영향을 조사하였다. 최고 제한속 도가 $5 \mathrm{mph}$ 증가한 주요 고속도로의 사망률은 $8 \%$ 증가하였고 기타 고속도로의 사망률은 $4 \%$ 증가하였 으며, 전체적으로 최고 제한속도가 증가하지 않는 경우에 비해 33,000 명의 교통 사망자가 더 많이 발생 하였다고 평가하였다.

본 연구에서는 경부선 천안IC-양재IC 구간의 교통 사고발생건수와 사고부상자수의 시계열 자료를 모형 화하고, 최고 제한속도 조정 전후 발생된 교통사고 변화의 분석을 통하여 제한속도 조정이 사고발생에 미친 영향을 연구한다. 또한 동일 기간의 타구간(부산IC-천안IC)을 비교구간으로 선정하고 두 구간의 교통사고 변화 차이를 이용하여 제한속도 상향조정 효과를 더 명확하게 판단하려고 한다.

본 논문의 구성은 다음과 같다. 2 장에서는 개입모형을 소개하고 3 장에서 천안IC-양재IC 구간에서 제한 속도 조정으로 인한 교통사고의 변화를 분석함과 동시에 천안IC-양재IC 및 부산IC-천안IC 두 구간의 교통사고 차이를 이용하여 제한속도 조정 효과를 분석한다. 제 4 장에서는 결과를 정리하였다.

\section{2. 개입모형}

시계열분석에서 정치 및 경제의 급격한 변화, 정책의 변화, 기업광고 전략의 교체나 근로자의 파업 등 과 같은 외부적 요인들이 기존의 시계열 자료에 영향을 주어 패턴에 변화가 생기는 경우, 이러한 사건 에 대하여 개입분석(intervention analysis)을 적용하여 자료에 미치는 영향을 모형화한다. Box와 Tiao (1975)는 여러 가지 정책들의 실행이 Los Angeles 지역의 오존 발생량에 주는 영향을 분석하기 위해 처 음으로 개입모형(intervention model)을 이용한 분석을 시도하였으며 두 가지 형태의 개입변수를 사용 하였다.

$T$ 시점에서 개입사건이 발생하여 그 효과가 발생시점 이후에도 지속적으로 영향을 미치는 경우 계단함 수(step function)로 표현하며 $T$ 시점에만 영향을 미치는 경우는 펄스함수(pulse function)로 표현한다.

$$
S_{t}{ }^{(T)}=\left\{\begin{array}{ll}
0, & t<T, \\
1, & t \geq T .
\end{array} \quad P_{t}^{(T)}= \begin{cases}0, & t \neq T, \\
1, & t=T .\end{cases}\right.
$$

식 (2.1)에 정의된 개입변수에 따라 개입요인의 효과는 여러 가지 형태로 변환될 수 있다. 개입효과가 $b$ 시차 후에 반영되고 그 크기가 점차로 줄어들거나 크기가 커지다가 새로운 수준으로 수렴하는 경우에 는 그 효과 $m_{t}$ 를 다음과 같이 표현할 수 있다.

$$
m_{t}=\frac{\omega}{1-\delta B} B^{b} S_{t}^{(T)}, \quad m_{t}=\frac{\omega}{1-\delta B} B^{b} P_{t}^{(T)} .
$$

단, $B$ 와 $b$ 는 각각 시차연산자(lag operator)와 지연시차(delay lag)를 나타내며 임의의 시계열 $Y_{t}$ 에 대하여 $B^{b} Y_{t}=Y_{t-b}$ 를 만족한다. $\omega$ 와 $\delta$ 는 각각 개입 초기효과 및 지속효과를 나타내는 모수이다. 식 (2.2)의 단일개입변수 형태는 $k$ 개의 개입들이 결합되어 동시에 시계열 자료에 반영되는 다중개입 
변수(multiple intervention variable)로 확장될 수 있다.

$$
\begin{aligned}
m_{t}= & \sum_{i=1}^{k} \frac{\omega_{i}(B) B^{b_{i}}}{\delta_{i}(B)} I_{i, t}, \\
& \omega_{i}(B)=\omega_{0}^{(i)}-\omega_{1}^{(i)} B-\cdots-\omega_{s_{i}}^{(i)} B^{s_{i}}, \quad \delta_{i}(B)=\delta_{0}^{(i)}-\delta_{1}^{(i)} B-\cdots-\delta_{r_{i}}^{(i)} B^{r_{i}} .
\end{aligned}
$$

단, $I_{i, t}$ 는 $S_{t}^{(T)}$ 또는 $P_{t}^{(T)}$ 와 같은 개입변수를 나타내며, $b_{i}, \omega_{i}(B)$ 와 $\delta_{i}(B)$ 는 각각 $i$ 번째 개입에 대 한 지연시차, 개입 초기효과 및 지속효과를 나타낸다. $s_{i}$ 와 $r_{i}$ 는 각각 $i$ 번째 개입효과에 대한 $\omega_{i}(B)$ 와 $\delta_{i}(B)$ 의 차수이다.

$T$ 시점에서 개입 $m_{t}$ 가 발생하였다고 가정하면 개입효과를 고려한 ARIMA 모형의 일반적인 형태는 다 음과 같이 나타낼 수 있다.

$$
Y_{t}=m_{t}+N_{t}
$$

외부개입이 있기 전의 사전개입모형(pre-intervention model)을 $\left\{N_{t}, t<T\right\}$ 로 표기한다. 사전개입모 형은 흔히 계절주기가 $s$ 인 $\operatorname{ARIMA}(p, d, q)(P, D, Q)_{s}$ 모형과 같은 승법계절모형(multiplicative autoregressive moving average model)이 주로 사용되며 다음과 같이 표현된다.

$$
\phi(B) \Phi\left(B^{s}\right)\left(1-B^{s}\right)^{D}(1-B)^{d} N_{t}=\theta_{0}+\theta(B) \Theta\left(B^{s}\right) \varepsilon_{t} .
$$

모형 $N_{t}$ 의 autoregressive (AR) 차수는 $p+P s$ 이며 moving average (MA) 차수는 $q+Q s$ 이고, $\Phi\left(B^{s}\right)$ 와 $\Theta\left(B^{s}\right)$ 는 각각 계절 $\mathrm{AR}$ 과 계절 $\mathrm{MA}$ 다항식, $\phi(B)$ 와 $\theta(B)$ 는 비계절 $\mathrm{AR}$ 과 $\mathrm{MA}$ 다항식이며 다음과 같 이 정의된다.

$$
\begin{aligned}
\theta(B) & =1-\theta_{1} B-\theta_{2} B^{2}-\theta_{q} B^{q}, \\
\phi(B) & =1-\phi_{1} B-\phi_{2} B^{2}-\phi_{p} B^{p}, \\
\Theta\left(B^{s}\right) & =1-\Theta B^{s}-\Theta_{2} B^{2 s}-\Theta_{Q} B^{Q s}, \\
\Phi\left(B^{s}\right) & =1-\Phi_{1} B^{s}-\Phi_{2} B^{2 s}-\Phi_{P} B^{P s} .
\end{aligned}
$$

일반적으로 개입모형에서 개입효과의 크기 $\xi_{t}$ 는 다음과 같이 개입 이전 시계열 $N_{t}$ 에 대한 개입 이후 시 계열 $Y_{t}$ 의 상대적인 변화량 $(\%)$ 으로 계산한다.

$$
\xi_{t}=\frac{Y_{t}-N_{t}}{N_{t}} \times 100=\frac{m_{t}}{N_{t}} \times 100 .
$$

\section{3. 경부고속도로 최고속도 상향에 따른 영향 분석}

\section{1. 분석자료}

본 연구에서의 교통사고 관련 자료는 경찰청과 도로교통공단에서 수집된 자료를 통합하여 제공하는 교 통사고분석시스템(Traffic Accident Analysis System; TAAS)을 활용하였다. 경부고속도로를 대상으 로 2007년 1월부터 2015년 5월까지의 총 101개월의 사고발생건수, 사고부상자수에 관한 월별자료를 수 집하고 제한속도의 상향효과를 분석하기 위하여 부산 기점을 기준으로 부산IC-천안IC $(0-341.14 \mathrm{~km})$ 와 천안IC-양재 $\mathrm{IC}(341.14-416.05 \mathrm{~km})$ 로 구분하였다.

개입분석에서 독립변수로 사용된 교통량 자료는 한국도로공사에서 제공되는 인터체인지간 월별데이터 를 활용하였다. 경부고속도로를 기준으로 서울, 수원신갈, 기흥, 오산, 안성, 천안 6 개의 고속도로 영업 소를 기초단위로 집계한 데이터이며 상행선 및 하행선의 양방향 전체 교통량 자료이다. 


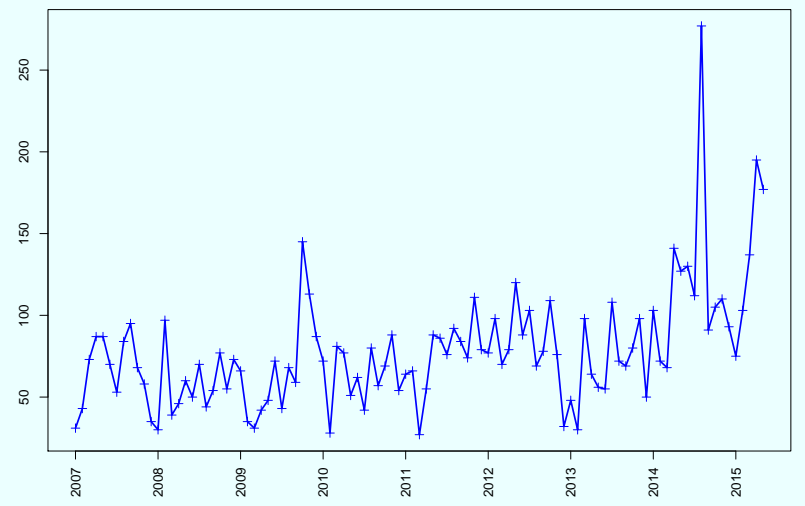

Figure 3.1. Time-series plot for the number of the traffic injuries from Jan 2007 to May 2015.

Table 3.1. Information criteria for potential pre-intervention models in case of the traffic injuries

\begin{tabular}{ccc}
\hline Model & AIC & BIC \\
\hline $\operatorname{ARIMA}(1,0,1)(0,1,0)_{s=12}$ & 45.9883 & 48.9198 \\
$\operatorname{ARIMA}(1,1,0)(2,1,1)_{s=12}$ & 53.5520 & 57.8540 \\
$\operatorname{ARIMA}(0,0,1)(2,1,0)_{s=12}$ & 54.4522 & 57.3202 \\
\hline
\end{tabular}

$\mathrm{AIC}=$ Akaike's information criterion; BIC $=$ Bayesian information criterion.

\section{2. 천안IC-양재IC 구간 분석}

3.2.1. 사고부상자수 2007년부터 2015년까지의 사고부상자수 관측자료의 시계열 그림은 Figure 3.1 과 같다. 사전개입모형의 식별은 2010 년 8 월까지(총 44 개월)의 시계열 자료를 사용하였다. 원자료 는 비정상성 시계열 자료로서 추세와 계절성이 존재하므로 자연로그변환과 1 차 또는 12 차 차분을 진행 하여 자료를 정상화하였으며 계절형과 비계절형 자기상관함수(autocorrelation function; $\mathrm{ACF}$ ), 부분 자기상관함수(partial autocorrelation function; PACF)을 토대로 모형의 적절한 차수를 결정하였다. 잠재모형을 선택하기 위하여 Akaike's information criterion (AIC)와 Bayesian information criterion (BIC) 값을 사용하였으며 Table 3.1 에서와 같이 3 개의 후보 모형을 선정하였다.

선택된 3 개의 모형을 추정하고 추정된 모형 모수들의 유의성 여부 및 잔차 진단을 통한 결과 최종 모형 으로 $\operatorname{ARIMA}(1,0,1)(0,1,0)_{s=12}$ 을 선택하였다. 특히, 모형의 진단 단계에서 잔차의 $\mathrm{ACF}$ 및 $\mathrm{PACF}$, 잔차의 포맨토우검정(portmanteau test)을 통하여 모든 시차에서 백색잡음(white noise) 성질을 만족 하는 것을 확인하였다. $Z_{1 \mathrm{t}}$ 가 원시계열로서 사고부상자수를 나타낸다고 할 때, 추정된 모형은 다음과 같다. 단, 괄호 안의 숫자는 추정량의 표준오차를 나타낸다.

$$
\begin{gathered}
(1-\phi B)\left(1-B^{12}\right) \ln \left(Z_{1 t}\right)=(1-\theta B) \varepsilon_{t} \\
\hat{\phi}=-0.6981(0.1949), \quad \hat{\theta}=-0.9436(0.0967) .
\end{gathered}
$$

사고부상자수는 2011년 4월부터 급격하게 증가하여 2011년 7월부터는 2010년 9월(개입시점) 이전 시 계열 자료와는 다른 흐름을 보이며, 2013년 2월부터는 변화의 폭이 점점 커지는 경향이 있다. 다만, 2012년 말과 2013년 초는 여전히 개입 이전과 비슷한 계절적 영향을 보이고 있다. 이를 모형에 반영하 기 위하여 2012년 12월과 2013년 2월 시점에 계절 더미변수를 적용하였으며, 이 시점의 사고부상자 감 소 원인을 명절효과 또는 계절효과로 판단하였다. 또한, 교통사고 시계열에 영향을 미칠 수 있는 외적 


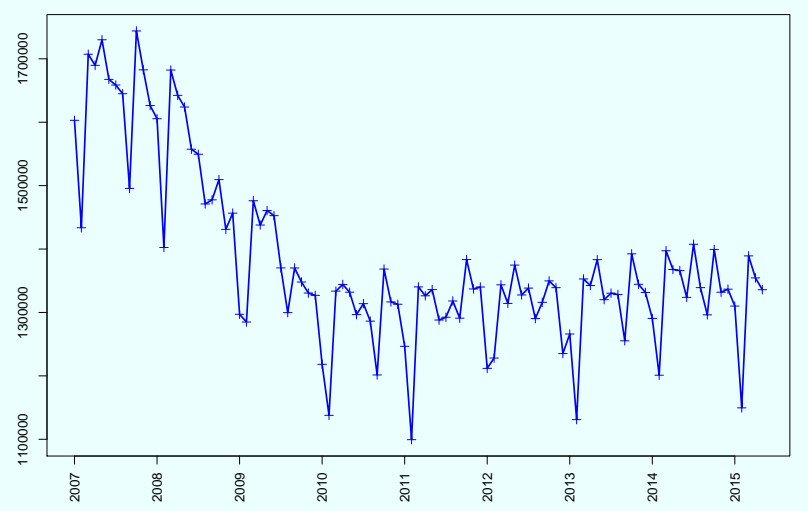

Figure 3.2. Time-series plot for the monthly traffic volume of the section of Cheonan IC-Yangjae IC from Jan 2007 to May 2015.

요인을 제거하고자 교통량 $X_{t}$ 를 외생(exogenous) 독립변수로 사용하였다. Figure 3.2 는 교통량의 시계 열 그림을 나타내며 사고부상자수의 계절성을 흐름을 잘 반영하고 있다. 단, 2010 년 이전까지는 다양한 경부고속도로의 교통망 확충 등으로 인하여 교통량이 급격하게 감소하고 있는 추세가 보인다. 사전개입 모형에 외생 변수를 추가한 모형은 다음과 같다.

$$
\begin{aligned}
\ln \left(Z_{1 t}\right)= & \beta_{1} X_{t}+\omega_{1} \mathrm{ADJ}_{1}+\omega_{2} \mathrm{ADJ}_{2}+\frac{(1-\theta B)}{(1-\phi B)\left(1-B^{12}\right)} \varepsilon_{t} \\
& \mathrm{ADJ}_{1}=\left\{\begin{array}{ll}
1, & t=\text { Dec.2012, } \\
0, & t \neq \text { Dec.2012, }
\end{array} \quad \mathrm{ADJ}_{2}= \begin{cases}1, & t=\text { Feb.2013, } \\
0, & t \neq \text { Feb.2013. }\end{cases} \right.
\end{aligned}
$$

개입의 형태는 제한속도 상향조정이 2010년 9월 이후 지속적으로 부상자 수에 영향을 미친다고 보아 계 단함수의 형태로 정의하였다. 개입효과는 제한속도 조정방안이 실시된 7 개월 후부터 반영되고, 이 기간 부터 시계열 수준에 변화를 가져왔다고 판단하였다.

$$
m_{t}=\frac{\omega_{0}}{1-\delta B} B^{7} S_{t}, \quad S_{t}=\left\{\begin{array}{l}
0, \quad t<\text { Sep.2010 } \\
1, \quad t \geq \text { Sep.2010 }
\end{array}\right.
$$

최종 개입모형은 개입이 있기 전의 자료에 적합한 모형을 반영하여 다음과 같으며 개입모형의 조건부 최 소제곱추정량(conditional least squares estimation)은 Table 3.2 와 같다.

$$
\ln \left(Z_{1 t}\right)=\frac{\omega_{0}}{1-\delta B} B^{7} S_{t}+\beta_{1} X_{t}+\omega_{1} \mathrm{ADJ}_{1}+\omega_{2} \mathrm{ADJ}_{2}+\frac{(1-\theta B)}{(1-\phi B)\left(1-B^{12}\right)} \varepsilon_{t} .
$$

추정된 개입변수와 계절조정변수들은 모두 유의하게 나타났고 개입효과는 전체 사고부상자수를 증가시 키는 방향으로 작용하였다. 다만, 개입 초기효과를 나타내는 $\omega_{0}$ 는 유의하지 않았으나 지속효과 $\delta$ 는 유 의하였으므로 개입변수를 그대로 유지하였다. 식 (3.4)와 같이 추정된 최종 개입모형을 사용하면, 경 부고속도로 최고 제한속도 상향으로 인한 효과를 계산할 수 있다 (Cryer과 Chan, 2008). 예를 들면, 2011년 4월 사고부상자수는 다음과 같은 계산을 통하여 $21.36 \%$ 증가하였고,

$$
\{\exp (0.1936)-1\} \times 100 \%=21.36 \%
$$


Table 3.2. Estimation results for the intervention model in case of the traffic injuries

\begin{tabular}{crcrrr}
\hline Parameter & Estimate & Standard error & $t$-value & Approx. $\operatorname{Pr}<|t|$ & Variable \\
\hline$\theta$ & 0.7756 & 0.1825 & 4.25 & $<0.0001$ & $Z_{1 \mathrm{t}}$ \\
$\phi$ & 0.9177 & 0.1254 & 7.32 & $<0.0001$ & $Z_{1 \mathrm{t}}$ \\
$\beta_{1}$ & -2.0222 & 1.1582 & -1.75 & 0.0850 & $X_{t}$ \\
$\omega_{1}$ & -0.7027 & 0.3313 & -2.12 & 0.0373 & $\mathrm{ADJ}_{1}$ \\
$\omega_{2}$ & -0.9971 & 0.3313 & -3.01 & 0.0036 & $\mathrm{ADJ}_{2}$ \\
$\omega_{0}$ & 0.1936 & 0.1361 & 1.42 & 0.1591 & $S_{t}$ \\
$\delta$ & 0.6926 & 0.2495 & 2.78 & 0.0070 & $S_{t}$
\end{tabular}

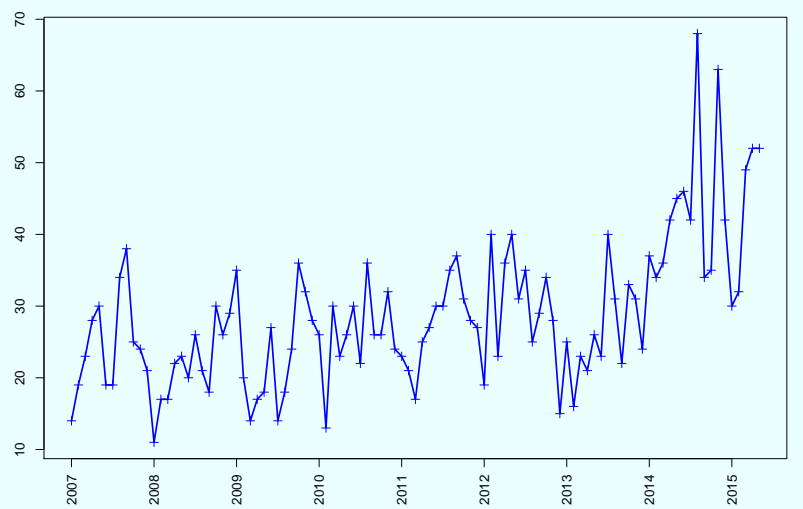

Figure 3.3. Time-series plot for the number of the traffic accidents from Jan 2007 to May 2015.

Table 3.3. Information criteria for potential pre-intervention models in case of the traffic accidents

\begin{tabular}{ccc}
\hline Model & AIC & BIC \\
\hline $\operatorname{ARIMA}(0,1,1)(0,1,1)_{s=12}$ & 35.2172 & 38.2103 \\
$\operatorname{ARIMA}(1,1,1)(0,1,1)_{s=12}$ & 35.9934 & 40.4829 \\
$\operatorname{ARIMA}(1,1,1)(0,1,0)_{s=12}$ & 37.9533 & 40.9463 \\
\hline
\end{tabular}

$\mathrm{AIC}=$ Akaike's information criterion; BIC = Bayesian information criterion.

2011 년 8월에는 $\left\{\exp \left(\omega_{0}+\omega_{0} \delta+\omega_{0} \delta^{2}+\cdots+\omega_{0} \delta^{4}\right)-1\right\} \times 100 \%=52.94 \%$ 만큼 증가하여 $50 \%$ 를 초 과하였다. 2012년 10 월이후부터 개입변수의 영향은 일정 수준으로 수렴하여 사고부상자수의 발생에 영 향을 미친다.

3.2.2. 사고발생건수 2007년 1 월부터 2015 년 5 월까지의 천안IC-양재IC 구간의 월별 사고발생건 수 시계열 그림은 Figure 3.3과 같다. 사고발생건수에 대한 개입효과를 분석하기 위하여 먼저 2007년 1 월부터 2010년 8월까지의 시계열 자료를 이용하여 사전개입모형을 구축하였다. 자료의 시계열 그림과 $\mathrm{ACF}, \mathrm{PACF}$ 의 상관도표를 기반으로 모형 식별 결과 Table 3.3 과 같은 후보모형을 선정하였다.

Table 3.3 의 3 가지 후보모형을 검토한 결과 다음과 같은 $\operatorname{ARIMA}(0,1,1)(0,1,1)_{s=12}$ 모형을 선택하였 다. 단, $Y_{1 t}$ 는 원시계열로서 사고발생건수를 나타낸다.

$$
\begin{gathered}
(1-B)\left(1-B^{12}\right) \ln \left(Y_{1 t}\right)=(1-\theta B)\left(1-\Theta B^{12}\right) \varepsilon_{t}, \\
\hat{\theta}=0.8225(0.1035), \quad \hat{\Theta}=0.4859(0.1956) .
\end{gathered}
$$


Table 3.4. Estimation results for the intervention model in case of the traffic accidents

\begin{tabular}{crcrrc}
\hline Parameter & Estimate & Standard error & $t$-value & Approx. Pr $<|t|$ & Variable \\
\hline$\theta$ & 0.6051 & 0.0963 & 6.28 & $<0.0001$ & $Y_{1 \mathrm{t}}$ \\
$\Theta$ & 0.7170 & 0.0940 & 7.63 & $<0.0001$ & $Y_{1 \mathrm{t}}$ \\
$\beta_{1}$ & -2.4144 & 1.0838 & -2.23 & 0.0289 & $X_{t}$ \\
$\omega_{1}$ & -0.6481 & 0.3059 & -2.12 & 0.0375 & ADJ $_{1}$ \\
$\omega_{0}$ & 0.1047 & 0.1297 & 0.81 & 0.4220 & $S_{t}$ \\
$\delta$ & 0.8102 & 0.3083 & 2.63 & 0.0140 & $S_{t}$ \\
\hline
\end{tabular}

또한, 3.1.1절의 사고부상자수와 유사하게 천안IC-양재IC 구간의 교통량 $X_{t}$ 와 계절 더미변수 $\mathrm{ADJ}_{1}$ 을 사용하여 사고발생건수에 미치는 외생적 요인 및 계절성을 모형에 추가하였다. 다음은 사전개입모형의 모형식이다.

$$
\begin{gathered}
\ln \left(Y_{1 t}\right)=\beta_{1} X_{t}+\omega_{1} \mathrm{ADJ}_{1}+\frac{(1-\theta B)\left(1-\Theta B^{12}\right)}{(1-B)\left(1-B^{12}\right)} \varepsilon_{t}, \\
\mathrm{ADJ}_{1}= \begin{cases}1, & t=\text { Dec. } 2012 \\
0, & t \neq \text { Dec.2012. }\end{cases}
\end{gathered}
$$

다음으로 제한속도 상향으로 인한 사고발생건수의 변화를 모형화하였다. 개입효과로는 다음과 같은 세 가지 형태를 고려하였다. 우선, 2010년 9월 개입시점을 기준으로 다음 달인 10 월부터 지속적으로 개입 영향을 미치는 경우 $\left(m_{t}^{(1)}\right)$ 를 고려할 수 있으며, 다음으로 개입이 지체되었다고 간주하고 자료의 평균수 준이 2011년 4월 또는 4 월과 5 월 모두부터 지수적으로 변화하기 시작하는 두가지 경우 $\left(m_{t}^{(2)}, m_{t}^{(3)}\right)$ 를 고려할 수 있다. $m_{t}^{(2)}$ 과 $m_{t}^{(3)}$ 의 개입 형태는 Cho 등 (2015) 또는 Kim과 Seong (2011)을 참고한다.

$$
m_{t}^{(1)}=\omega_{0} B S_{t}, \quad m_{t}^{(2)}=\frac{\omega_{0}}{1-\delta B} B^{7} S_{t}, \quad m_{t}^{(3)}=\frac{\omega_{0}-\omega_{1} B}{1-\delta B} B^{7} S_{t}
$$

위의 3 가지 개입모형을 적합시킨 결과 $m_{t}^{(1)}$ 은 유의하지 않았으며 $m_{t}^{(3)}$ 는 $\omega_{1}$ 항이 유의하지 않았다. 따 라서, $m_{t}^{(2)}$ 에 의한 개입변수가 가장 적절하다고 판단하였으며, 사전개입모형과 개입변수 $m_{t}^{(2)}$ 를 포함한 최종 개입모형은 다음과 같다.

$$
\ln \left(Y_{1 t}\right)=\frac{\omega_{0}}{1-\delta B} B^{7} S_{t}+\beta_{1} X_{t}+\omega_{1} \mathrm{ADJ}_{1}+\frac{(1-\theta B)\left(1-\Theta B^{12}\right)}{(1-B)\left(1-B^{12}\right)} \varepsilon_{t} .
$$

사고발생건수에 대한 개입모형 추정은 조건부 최소제곱추정방법을 사용하였으며 Table 3.4 와 같다. 개 입변수와 계절조정변수들은 모두 유의하게 나타났고 개입효과는 전체 사고발생건수를 증가시키는 방향 으로 작용하였다.

사고발생건수에 대한 제한속도의 영향을 살펴보면 2011년 4월 사고발생건수는 $\{\exp (0.1047)-1\} \times$ $100=11.03 \%$ 만큼 증가하였고, 2012 년 3 월에는 $\left\{\exp \left(\omega_{0}+\omega_{0} \delta+\omega_{0} \delta^{2}+\cdots+\omega_{0} \delta^{11}\right)-1\right\} \times 100 \%=$ $50.74 \%$ 증가하여 $50 \%$ 를 초과하였으며 2013년 12 월이후부터는 일정 수준으로 수렴하였다.

Figure 3.4 는 사고부상자수 $\left(Z_{1 t}\right)$ 와 사고발생건수 $\left(Y_{1 t}\right)$ 에 영향을 미친 개입효과의 크기 $\xi_{t}$ 를 연도별로 보여준다. 제한속도 조정이 실시된 후 사고부상자수와 사고발생건수는 모두 증가하였으며 사고부상자 수에 미치는 개입효과는 사고발생건수보다 짧은 기간 내에 더 높은 수준으로 수렴하였다. 즉 최고 제한 속도의 증가에 따라 사고발생시 상해사고의 위험성이 더욱 커졌다고 판단할 수 있다. 

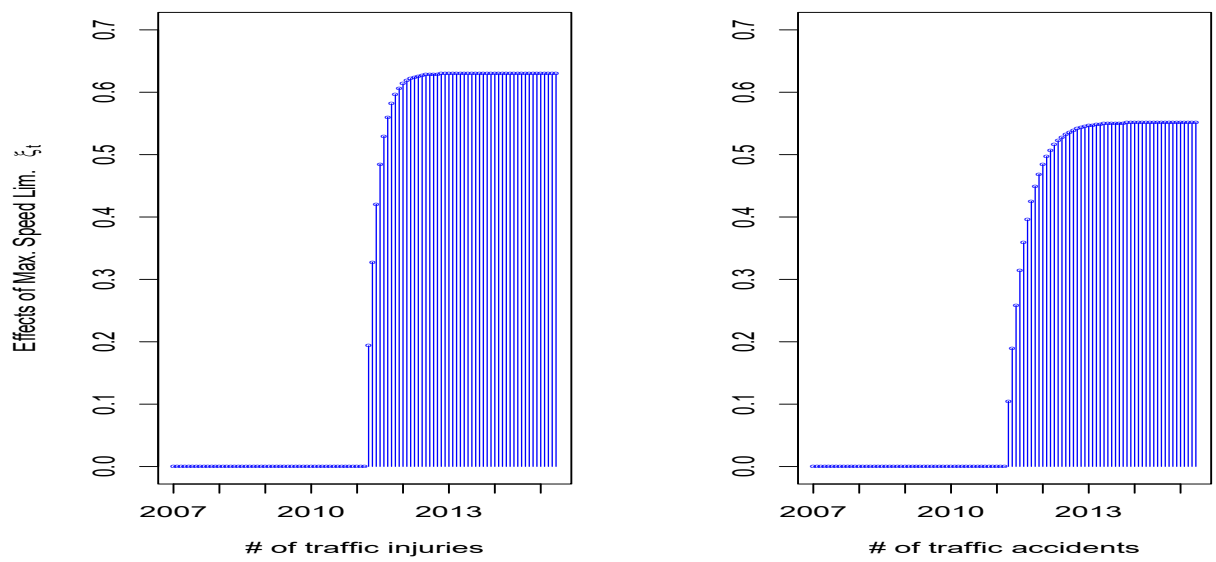

Figure 3.4. Effect sizes of the maximum speed limit on the traffic accidents and injuries.

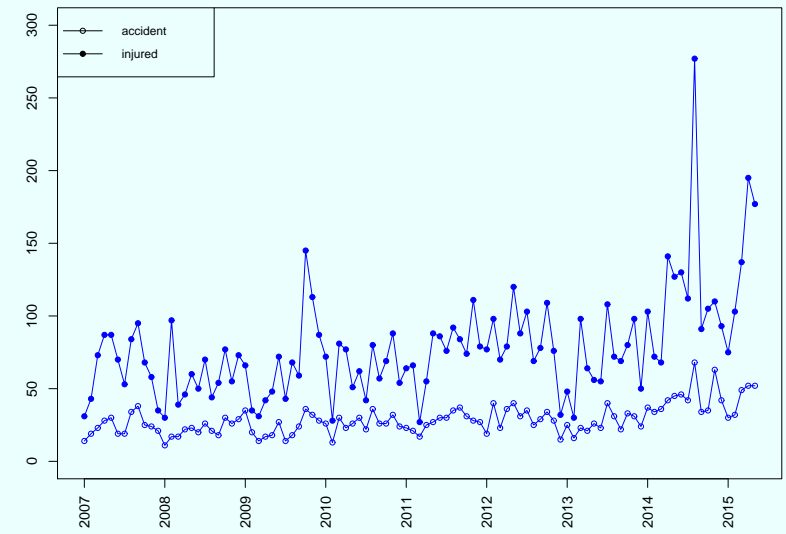

Figure 3.5. Time-series plot for the numbers of the traffic accidents and injuries of the section of Cheonan IC-Yangjae IC.

\section{3. 천안IC-양재IC 구간과 부산IC-천안IC 구간 차이 분석}

본 절에서는 천안IC-양재IC 구간에서만 시행되고 있는 최고 제한속도 변화의 개입 효과를 좀더 분명하 게 살펴보기 위하여, 부산IC-천안IC 구간과의 차이를 이용한다. 다양한 외부적 환경이 존재할 수 있으 므로 두 구간의 교통사고 시계열 자료의 차이를 단순히 최고 제한속도를 원인으로 간주하는 것은 쉽지 않다. 그러나, 본 연구에서 분석하는 시계열 자료 기간(2007년-2015년) 동안 두 구간의 교통사고 시계 열 자료에 서로 다른 영향을 미칠 수 있는 주요 변수는 최고 제한속도 변화 이외에는 찾기가 어려웠다. 본 연구에서는 최고 제한속도 이외에는 천안IC-양재IC와 부산IC-천안IC의 두 구간이 비슷한 외부적 환경 하에서 운영된다고 가정하고 제한속도의 상향조정 효과를 분석하려고 한다.

Figures 3.5 와 3.6 은 천안IC-양재IC 구간과 부산IC-천안IC 구간의 사고부상자수와 사고발생건수를 각 각 보여주고 있다. 천안IC-양재IC 구간의 사고부상자수와 사고발생건수는 증가하는 추세가 있지만 부 산IC-천안IC 구간은 반대로 감소하는 추세를 보인다. 


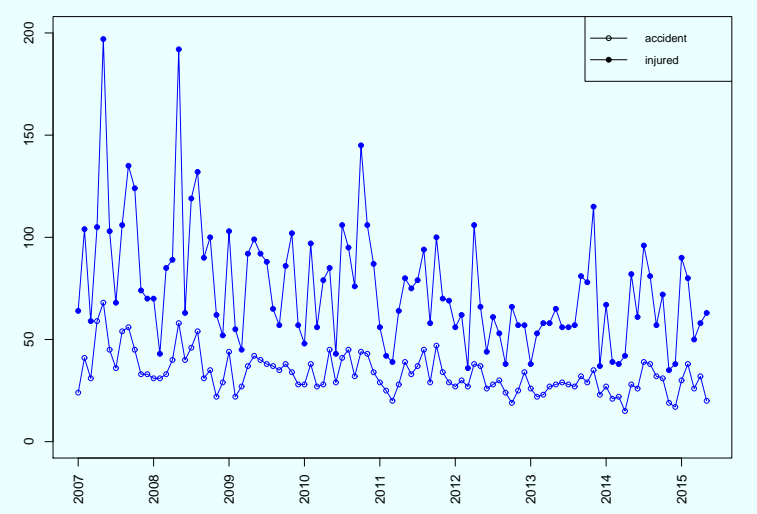

Figure 3.6. Time-series plot for the numbers of the traffic accidents and injuries of the section of Busan ICCheonan IC.

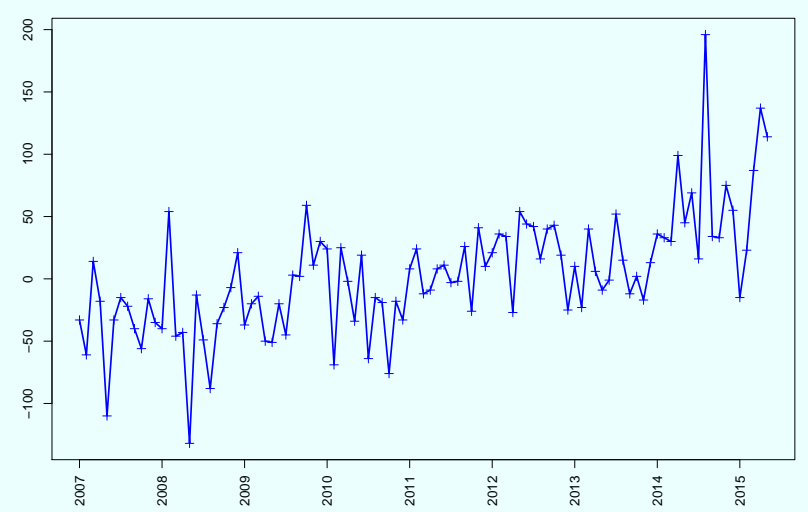

Figure 3.7. Time-series plot for the difference in the number of injuries of the two sections: Cheonan IC-Yangjae IC and Busan IC?Cheonan IC.

3.3.1. 사고부상자수 Figure 3.7 은 두 구간의 사고부상자수 차이를 나타낸 시계열 그림이다. 사고 부상자수 차이는 다음과 같이 $Z_{2 t}$ 로 정의하였다.

$$
\ln \left(Z_{2 t}\right)=\ln \left(Z_{1 t}^{\text {Major }}\right)-\ln \left(Z_{1 t}^{\text {Minor }}\right) .
$$

단, $Z_{1 t}^{\mathrm{Major}}$ 와 $Z_{1 t}^{\mathrm{Minor}}$ 는 각각 천안IC-양재 $\mathrm{IC}$ 구간과 부산IC-천안IC 구간의 사고부상자수를 나타내며, $Z_{2 t}$ 는 두 구간 사고부상자수의 비율(ratio)을 나타낸다. 2007년 1월부터 2010년 8월까지의 두 구간 사 고부상자수 차이 $\ln \left(Z_{2 t}\right)$ 를 이용하여 다음과 같은 사전개입모형 $\operatorname{ARIMA}(0,1,1)(0,1,1)_{s=12}$ 을 구축하 였다.

$$
\begin{gathered}
\left(1-B^{12}\right)(1-B) \ln \left(Z_{2 t}\right)=(1-\theta B)\left(1-\Theta B^{12}\right) \varepsilon_{t} \\
\hat{\theta}=0.8908(0.0900), \quad \hat{\Theta}=0.6716(0.2142) .
\end{gathered}
$$

두 구간의 차이를 이용한 최고 제한속도 효과를 분석하기 위하여 계절 더미변수는 서로 상쇄된다고 간 주하고 따로 고려하지 않았다. 개입효과가 반영된 시점은 제한속도가 상향된 시점에서 3 개월 지연되어 
Table 3.5. Estimation results for the intervention model based on the differenced traffic injuries of the two sections

\begin{tabular}{ccccc}
\hline Parameter & $\omega_{0}$ & $\delta$ & $\theta$ & $\Theta$ \\
\hline Estimate & 0.0939 & 0.8212 & 0.8359 & 0.5960 \\
Standard error & 0.1223 & 0.2834 & 0.0632 & 0.0980 \\
\hline
\end{tabular}

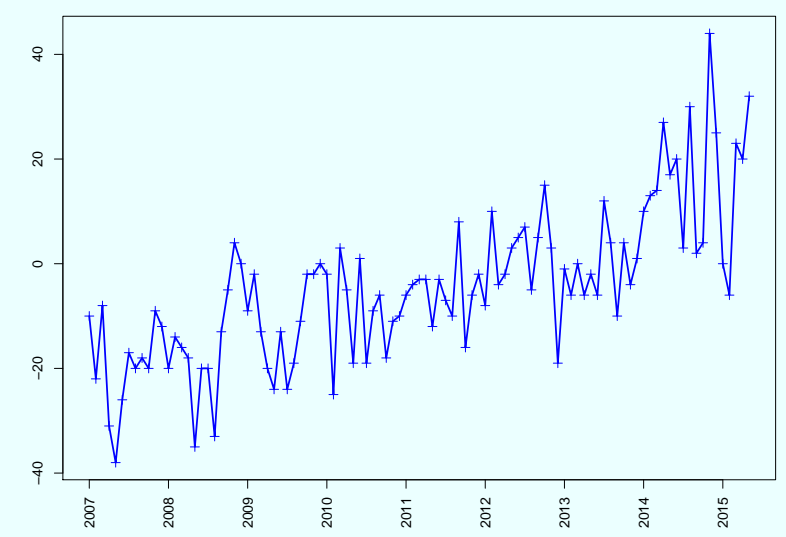

Figure 3.8. Time-series plot for the difference in the number of accidents of the two sections: Cheonan IC-Yangjae IC and Busan IC-Cheonan IC.

2010 년 12 월부터 사고부상자수의 발생에 영향을 미치는 것으로 고려하고 다음과 같은 개입변수 $m_{t}$ 를 정의하였다.

$$
m_{t}=\frac{\omega_{0}}{1-\delta B} B^{3} S_{t}
$$

개입변수를 포함한 시계열모형은 사전개입모형을 반영하여 다음과 같으며, 적합결과 추정된 모수들은 Table 3.5 와 같다. 개입효과를 포함한 각 모수들은 유의하고 사고부상자의 발생을 증가시키는 방향으로 반영되었다.

$$
\ln \left(Z_{2 t}\right)=m_{t}+N_{t}=\frac{\omega_{0}}{1-\delta B} B^{3} S_{t}+\frac{(1-\theta B)\left(1-\Theta B^{12}\right)}{(1-B)\left(1-B^{12}\right)} \varepsilon_{t} .
$$

개입효과가 처음으로 나타난 2010년 12 월에는 사고부상자수 차이가 $\{\exp (0.0939)-1\} \times 100=$ $9.38 \%$ 만큼 증가하였으며 2012 년 3월에는 $\left\{\exp \left(\omega_{0}+\omega_{0} \delta+\omega_{0} \delta^{2}+\cdots+\omega_{0} \delta^{15}\right)-1\right\} \times 100 \%=50.26 \%$ 만 큼 증가하였다 $\left(\omega_{0}=0.0939, \delta=0.8212\right) .2014$ 년 6 월에는 $\left\{\exp \left(\omega_{0}+\omega_{0} \delta+\omega_{0} \delta^{2}+\cdots+\omega_{0} \delta^{42}\right)-1\right\} \times$ $100 \%=52.50 \%$ 만큼 증가하여 6 월 이후부터는 점차 새로운 수준으로 수렴하였다.

3.3.2. 사고발생건수 Figure 3.8 은 두 구간의 사고발생건수 차이를 나타낸 시계열 그림이다. 사고 발생건수 차이는 다음과 같이 $Y_{2 t}$ 로 정의하였다.

$$
\ln \left(Y_{2 t}\right)=\ln \left(Y_{1 t}^{\text {Major }}\right)-\ln \left(Y_{1 t}^{\text {Minor }}\right)
$$

단, $Y_{1 t}^{\mathrm{Major}}$ 와 $Y_{1 t}^{\mathrm{Minor}}$ 는 각각 천안IC-양재IC 구간과 부산IC-천안IC 구간의 사고발생건수를 나타낸다. 먼저 2007년 1 월부터 2010 년 8월까지의 자료를 이용하여 다음과 같은 $\operatorname{ARIMA}(0,0,0)(0,1,1)_{s=12}$ 의 
Table 3.6. Estimation results for the intervention model based on the differenced traffic accidents of the two sections

\begin{tabular}{ccc}
\hline Parameter & $\omega_{0}$ & $\Theta$ \\
\hline Estimate & 0.0193 & 0.7311 \\
Standard error & 0.0029 & 0.0936 \\
\hline
\end{tabular}
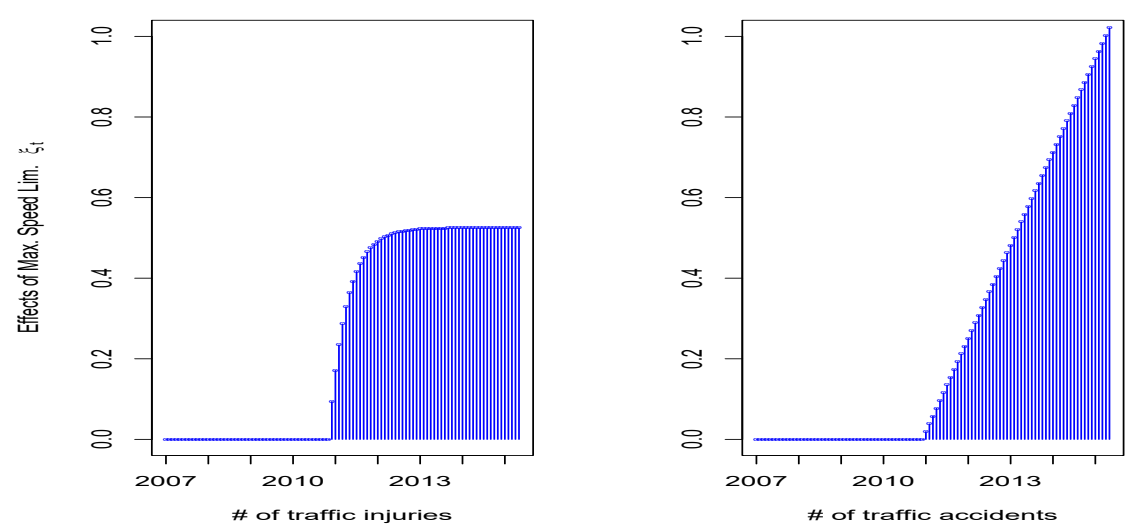

Figure 3.9. Effect sizes of the maximum speed limit on the traffic accidents and injuries based on the differences of the two sections: Cheonan IC-Yangjae IC and Busan IC-Cheonan IC.

사전개입모형을 적합하였다.

$$
\left(1-B^{12}\right) \ln \left(Y_{2 t}\right)=\left(1-\Theta B^{12}\right) \varepsilon_{t}, \quad \hat{\Theta}=0.7418(0.2264) .
$$

개입효과는 다음과 같이 두 가지 형태로 설정하였다. 2010 년 9 월 개입시점을 기준으로 4 시차 지연되어 다음해 1 월부터 자료에 지속적으로 영향을 미친다고 판단되어 개입효과의 크기가 점차로 증가하는 경우 인 $m_{t}^{(1)}$ 과 개입효과가 선형으로 증가하는 경우인 $m_{t}^{(2)}$ 는 다음과 같다.

$$
m_{t}^{(1)}=\frac{\omega_{0}}{1-\delta B} B^{4} S_{t}, \quad m_{t}^{(2)}=\frac{\omega_{0}}{1-B} B^{4} S_{t} .
$$

그러나, $m_{t}^{(1)}$ 의 적합은 유의하였으나 추정된 지속효과의 크기가 $\hat{\delta}=0.9939$ 로서 단위근 $\delta=1$ 임을 암시 하였으며 $m_{t}^{(2)}$ 의 적합은 $m_{t}^{(1)}$ 의 적합보다 $\mathrm{AIC}$ 와 $\mathrm{BIC}$ 를 더욱 감소시켰다(AIC: $88.07 \rightarrow 86.11$, BIC: $95.36 \rightarrow 91.00)$. 따라서, 사전개입모형에 개입변수 $m_{t}^{(2)}$ 를 적용한 다음과 같은 최종 개입모형을 적합 하였다.

$$
\ln \left(Y_{2 t}\right)=\frac{\omega_{0}}{1-B} B^{4} S_{t}+\frac{\left(1-\Theta B^{12}\right)}{\left(1-B^{12}\right)} \varepsilon_{t} .
$$

식 (3.14)의 모수 추정결과는 Table 3.6 과 같다. 조건부 최소제곱 추정량에 의한 결과 개입변수를 포함 한 모형의 모수는 모두 유의한 것으로 나타났다.

개입효과가 처음으로 나타난 2011 년 1월에는 사고발생건수가 $\{\exp (0.0193)-1\} \times 100 \%=1.95 \%$ 만큼 증가하였으며 2013년 2 월에는 $\{\exp (0.0193 \times 26)-1\} \times 100=65.17 \%$ 만큼 증가하였다. 사고발생건 수에 미치는 개입효과는 선형으로 증가하는 추세를 나타낸다. Figure 3.9 는 천안IC-양재IC와 부산IC천안IC 두 구간의 사고부상자수 차이와 사고발생건수 차이에 미친 개입효과의 크기 $\xi_{t}$ 를 연도별로 보여 준다. 
두 구간의 차이를 이용하여 교통사고에 반영된 개입효과를 살펴보면 2010 년 12 월부터 제한속도의 상향 조정은 사고부상자수의 변화에 반영되고 2012년 이후 그 추세는 점차로 수렴하지만, 사고발생건수는 개 입의 영향이 선형적으로 증가하는 추세를 나타낸다. 천안IC-양재IC와 부산IC-천안IC 두 구간의 차이 를 통해서 살펴볼 때, 최고 제한속도의 상향은 교통 사고부상자수에는 3.2 절과 유사한 결론을 내릴 수 있으나 교통 사고발생건수에는 심각한 영향을 미치고 있다고 판단된다.

\section{4. 결론}

본 논문에서는 경부고속도로 최고 제한속도 증가가 교통사고에 미친 영향을 분석하기 위하여 개입모형 을 적용하여 외부적 요인이 교통사고발생에 미치는 영향을 고려하여 두 가지 방법으로 효과분석을 진행 하였다. 제한속도 상향 후 교통 사고발생건수와 사고부상자수는 2011년 4월부터 모두 증가하는 추세이 며 특히 제한속도가 예전과 변화 없는 구간에 비하여 사고발생건수와 사고부상자수가 늘어나고 있는 추 세로 판단된다. 또한 제한속도 상향은 시차는 있으나 지속적으로 사고발생건수와 사고부상자수에 영향 을 미치는 것으로 나타났으며 2013년 이후부터는 제한속도 상향의 효과는 점차적으로 수렴하는 추세로 나타났다. 다만, 교통사고의 발생은 계절성 및 외부적 요인으로 인한 변동이 클 수 있기 때문에 이러한 외부적 요인을 설명할 수 있는 추가적인 변수를 분석해 보아야 할 필요가 있다. 또한 스마트폰의 사용, $\mathrm{DMB}$ 시청 등 전방주시 태만으로 발생된 교통사고에 관한 추가적인 연구가 필요하다.

\section{References}

Box, G. E. P. and Tiao, G. C. (1975). Intervention analysis with applications to economic and environmental problems, Journal of the American Statistical Association, 70, 70-79.

Cho, S., Son, Y., and Seong, B. (2015). Time Series Analysis Using SAS/ETS, Yulgokbooks, Seoul.

Cryer, J. D. and Chan, K. S. (2008). Time Series Analysis: With Applications in R, Springer-Verlag.

Farmer, C. M. (2016). Relationship of traffic fatality rates to maximum state speed limits, Traffic Injury Prevention, 18, 375-380.

Friedman, L. S., Barach, P., and Richter, E. D. (2007). Raised speed limits, case fatality and road deaths: a six-year follow-up using ARIMA models, Injury Prevention, 13, 156-161.

Kim, S. and Seong, B. (2011). Intervention analysis of Korea tourism data, The Korean Journal of Applied Statistics, 24, 735-743.

Lee, J. (2011). Effect analysis of the speed limit increase test operation on Gyeongbu Expressway (Technical report), Korea Expressway Corporation.

Ossiander, E. M. and Cummings, P. (2002). Freeway speed limits and traffic fatalities in Washington State, Accident Analysis and Prevention, 34, 13-18. 


\title{
경부고속도로 최고제한속도 상향에 따른 교통사고 영향 분석
}

\author{
송은화 $^{a} \cdot$ 성병찬 $^{a, 1}$ \\ ${ }^{a}$ 중앙대학교 응용통계학과
}

(2017년 7월 27일 접수, 2017년 8월 19일 수정, 2017년 8월 21일 채택)

요 약

본 논문에서는 개입모형(intervention model)을 이용하여 경부고속도로(천안IC-양재IC) 구간의 최고 제한속도 상 향 조정으로 인한 교통사고 발생의 영향을 분석하고자 하였다. 또한 천안IC-양재IC 구간과 부산IC-천안IC 구간의 차이를 이용하여 제한속도의 상향조정 효과를 분석하여 외부적 요인의 영향을 고려하지 않을 경우 제한속도 조정으 로 인한 교통사고의 변화를 분석하였다. 그 결과, 제한속도 상향 후 교통 사고발생건수와 사고부상자수는 모두 증가 하는 추세이며 특히 최고속도가 예전과 변화 없는 구간에 비교할 때 그 증가 추세는 더 심각한 것으로 판단된다.

주요용어: 개입분석, ARIMA 모형, 교통 사고발생건수, 교통 사고부상자수

이 논문은 2016 년도 정부(교육부)의 재원으로 한국연구재단의 지원을 받아 수행된 기초연구사업임 (No. NRF2016R1D1A1B03934857).

${ }^{1}$ 교신저자: (06974) 서울시 동작구 흑석로 84 , 중앙대학교 경영경제대학 응용통계학과.

E-mail: bcseong@cau.ac.kr 